\title{
Fabrication of Large Size Ex Vivo-Produced Oral Mucosal Equivalents for Clinical Application
}

\author{
Hiroko Kato, DDS, PhD, ${ }^{1,2}$ Cynthia L. Marcelo, PhD, James B. Washington, BS, ${ }^{1}$ \\ Eve L. Bingham, BS, and Stephen E. Feinberg, DDS, MS, PhD ${ }^{1}$
}

\begin{abstract}
The soft tissue reconstruction of significant avulsed and/or surgically created tissue defects requires the ability to manufacture substantial soft tissue constructs for repair of the resulting wounds. In this study, we detail the issues that need to be addressed in upsizing the manufacture of larger tissue-engineered devices (ex vivoproduced oral mucosa equivalent [EVPOME]) in vitro from a methodology previously used for smaller constructs. The larger-sized EVPOME, consisting of autologous human oral keratinocytes and a dermal substitute, AlloDerm ${ }^{\circledR}$, was fabricated for the purpose of reconstructing large clinical defects. Regulated as an autologous somatic cell therapy product, the fabrication process abided by current Good Manufacturing Practices and current Good Tissue Practices as required by the Center for Biologics Evaluation and Research (CBER) of the United States Food and Drug Administration (FDA). Successful fabrication of large EVPOMEs utilized a higher cell seeding density $\left(5.3 \times 10^{5}\right.$ cells $\left./ \mathrm{cm}^{2}\right)$ with a relatively thinner AlloDerm, ranging from 356.6 to $508.0 \mu \mathrm{m}$ in thickness. During the air-liquid interface culture, the thickness of the scaffold affected the medium diffusion rate, which, in turn, resulted in changes of epithelial stratification. Histologically, keratinocyte progenitor (p63), proliferation (Ki-67), and late differentiation marker (filaggrin) expression showed differences correlating with the expression of glucose transporter-1 (GLUT1) in the EVPOMEs from the thickest (550$1020 \mu \mathrm{m})$ to the thinnest $(228.6-330.2 \mu \mathrm{m})$ AlloDerm scaffold. Glucose consumption and 2-deoxyglucose (2DG) uptake showed direct correlation with scaffold thickness. The scaffold size and thickness have an impact on the cellular phenotype and epithelial maturation in the manufacturing process of the EVPOME due to the glucose accessibility influenced by the diffusion rate. These outcomes provide basic strategies to manufacture a large-sized, healthy EVPOME graft for reconstructing large mucosa defects.
\end{abstract}

\section{Introduction}

$\mathbf{R}$ ECONSTRUCTIVE PROCEDURES OF the oral cavity often result in open wounds in the mouth, requiring the need for mucosal tissue for intraoral reconstructive surgery. A free mucosal graft used for reconstruction or closure of the open wounds can prevent microbial infection, excessive fluid loss, and foreign material contamination or relapse secondary to wound contracture. Oral mucosa and skin grafts, both requiring the harvesting of tissue from a second surgical site, can result in increased patient morbidity. ${ }^{1}$ In addition, oral mucosa is limited in supply, while splitthickness skin grafts, although more easily available, contain adnexal structures and express a different pattern of keratinization. ${ }^{2}$ Thus, clinical needs for regeneration of oral soft tissue remain to be solved.

Previously, we reported a clinical trial of the ex vivoproduced oral mucosa equivalent (EVPOME) device that demonstrated safety for intraoral grafting and that validated its use to augment the development of keratinized tissue around teeth. ${ }^{3,4} \mathrm{~A}$ circular piece of EVPOME $20 \mathrm{~mm}$ in diameter $\left(3.14 \mathrm{~cm}^{2}\right)$ was sufficient for small areas of periodontal procedures; however, a larger EVPOME graft is necessary for tumor, trauma, and other potential reconstructive surgical procedures requiring significant amounts of soft tissue. In fact, for resurfacing larger defects, we need to manufacture multiple circular pieces of $3.14 \mathrm{~cm}^{2}$ EVPOMEs due to few product lines of cell culture. ${ }^{3,4}$ The size of the $3 \times 5 \mathrm{~cm}$ EVPOME has been manufactured under the investigator-initiated phase I/II clinical trial for vestibuloplasty at the University of Michigan. The size of $3 \times 5 \mathrm{~cm}$ was selected because it is sufficient to cover alveolar mucosa for the placement of several dental implants.

Scaffold design is well investigated in tissue engineering. The optimal design to provide a suitable microenvironment for cells varies with cell type, culture condition, and material. ${ }^{5}$

\footnotetext{
${ }^{1}$ Department of Oral and Maxillofacial Surgery, University of Michigan Health System, Ann Arbor, Michigan.

${ }^{2}$ Course for Oral Life Science, Department of Biomimetics, Postgraduate School of Medical and Dental Sciences, Niigata University, Niigata, Japan.
} 
AlloDerm ${ }^{\circledR}$ (LifeCell, Branchburg, NJ) is an acellular human dermal matrix having structures of polarity and porosity. Various thicknesses of AlloDerm are commercially available; however, the effect of varying thicknesses on the fabrication of tissue-engineered mucosa/skin still remains unclear.

Glucose consumption of EVPOMEs was successfully used as a quality control test in the last clinical trial as it reflects the viable cell number of the product. ${ }^{3,6}$ Although glucose metabolism of keratinocytes is important for epithelial proliferation and differentiation, there are few studies investigating the effects of glucose on keratinocytes in a three-dimensional culture condition, ${ }^{7,8}$ while several tissueengineered oral mucosa constructs have been developed for a disease model, evaluation of drug delivery systems, and clinical transplantation. ${ }^{9-11}$

The aim of this study is the fabrication of a $15 \mathrm{~cm}^{2}$ EVPOME for reconstruction of significant soft tissue defects. Parametrics examined include the effects of the scaffold thickness on cellular phenotype, the epithelial maturation of the keratinocytes, and glucose metabolism.

\section{Materials and Methods}

\section{Procurement of human oral mucosa}

Keratinized oral mucosa was obtained from eight patients; three males and five females with a mean age of 42.5 years without any malignancies undergoing tooth extraction and/or minor dentoalveolar surgery. The protocol for harvesting human oral mucosal tissue was approved by a University of Michigan Internal Review Board.

\section{Primary oral keratinocytes and serial cultures}

As described previously, with modification, ${ }^{12}$ mucosal tissue was digested overnight with $0.04 \%$ trypsin solution (Life Technologies, Carlsbad, CA) with $19.25 \mu \mathrm{g} / \mathrm{mL}$ of gentamicin (Life Technologies) and $0.765 \mu \mathrm{g} / \mathrm{mL}$ of fungizone (Life Technologies) at room temperature and transferred into $0.0125 \%$ defined trypsin inhibitor (DTI; Life Technologies) to neutralize the trypsin solution. Oral keratinocytes were mechanically dissociated from the submucosal connective tissue, collected, and centrifuged. Dissociated oral keratinocytes were resuspended in a chemically defined culture system $\left(\right.$ EpiLife $^{\circledR}$, supplemented with Epilife defined growth supplement (EDGS) and $0.06 \mathrm{mM}$ calcium; Life Technologies) and seeded into one T-25 flask (Corning, Corning, NY). Ambient oxygen in an incubator at $37^{\circ} \mathrm{C}$ in a humidified $5.0 \% \mathrm{CO}_{2}$ environment was used for following the culture procedure. For the serial cultures, cells were de- tached in $0.025 \%$ trypsin/EDTA (Life Technologies) and neutralized by DTI. P2-P4 were used for the experiments.

\section{Cultureware design and fabrication of large size EVPOME}

We designed a $30 \times 50 \times 15 \mathrm{~mm}$ corral and a $95 \mathrm{~mm}$ diameter $\times 5 \mathrm{~mm}$ height waffle grid made of an acrylonitrilebutadiene-styrene (ABS) plastic, which were made by University of Michigan 3D Laboratory using fused deposition modeling with a Dimension Elite FDM 3D Printer (Stratasys, Eden Prairie, MN). EVPOMEs were fabricated as previously reported with modifications of cell number and size. ${ }^{3}$ A corral was put on the inside of a $100-\mathrm{mm}$ culture dish (Corning) and glued on the dish with Indermil ${ }^{\circledR}$ (n-butylcynoacrylate) tissue adhesive (Henkel KgaA, Düsseldorf, Germany). Once the corrals were glued in place, a $3 \times 5 \mathrm{~cm}$ piece of AlloDerm, presoaked with human type IV collagen $\left(5 \mu \mathrm{g} / \mathrm{cm}^{2}\right)$ (Sigma-Aldrich, St. Louis, MO), was placed inside of the corral composite. P2-P4 oral mucosa keratinocytes with the seeding densities of $6.6 \times 10^{5}$, $5.3 \times 10^{5}, 4.0 \times 10^{5}$, and $2.0 \times 10^{5}$ cells $/ \mathrm{cm}^{2}$ were seeded onto the AlloDerm and cultured submerged for a day with $20 \mathrm{~mL}$ of the medium. Then, the cell-seeded AlloDerm was moved to another dish with an unglued corral, which permitted medium perfusion to the AlloDerm, but did not allow the AlloDerm to move or float in the medium. Following 3 days in a submerged culture under the same condition as the primary cell culture fed with $60 \mathrm{~mL}$ of the medium, which was changed every day, the construct was raised to an airliquid interface for an additional 7 days using a complex comprising a waffle grid and a $75-\mathrm{mm}$ transwell membrane insert (Corning) in a 150-mm culture dish (Corning) fed with $60 \mathrm{~mL}$ of the medium, which was changed every other day (Fig. 1).

Besides the $3 \times 5 \mathrm{~cm}$ EVPOME, two different sizes of the EVPOME $\left(0.75\right.$ and $\left.1.75 \mathrm{~cm}^{2}\right)$ routinely fabricated in our laboratory were identically cultured for comparison. A 48well plate was used for the $0.75 \mathrm{~cm}^{2}$ EVPOME during submerged culture, and a 12-well plate was used for the $1.75 \mathrm{~cm}^{2}$ EVPOME. For air-liquid culture, a $75-\mathrm{mm}$ transwell was used for either condition. EpiLife supplemented with EDGS and $1.2 \mathrm{mM}$ calcium was used during the entire manufacture of the EVPOME devices.

\section{Optimization of cell seeding density by histology and MTT assay}

Four seeding densities stated above were tested for the fabrication of the $3 \times 5 \mathrm{~cm}$ EVPOME. Day 11 EVPOME was

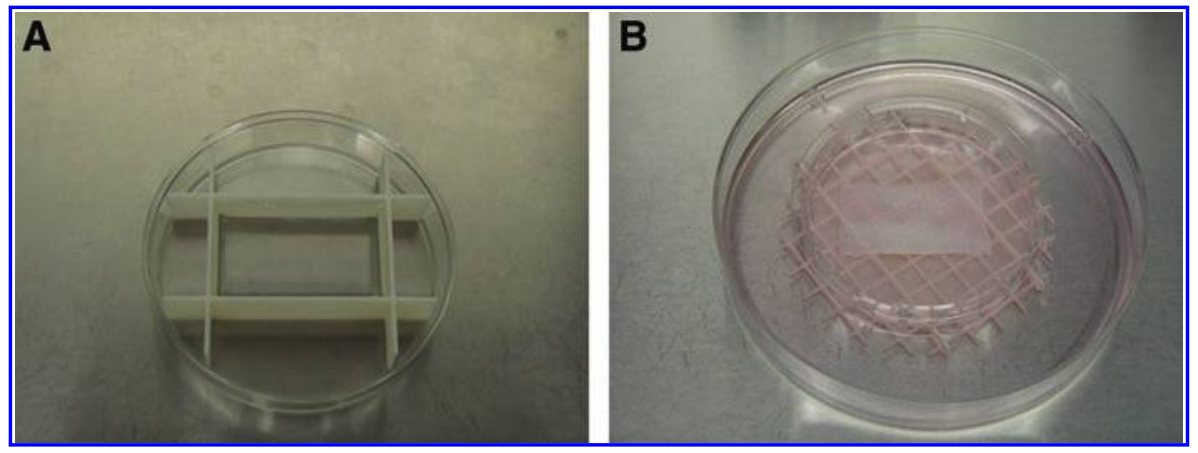

FIG. 1. Culture devices and procedure of large ex vivo-produced oral mucosa equivalent (EVPOME). (A) Glued corral on the 100-mm culture dish using Indermil $^{\circledR}$. (B) Air-liquid interface culture using a $150-\mathrm{mm}$ dish with waffle and a transwell insert from days 4 to 11 . Color images available online at www.liebertpub $. \mathrm{com} / \mathrm{tec}$ 
analyzed by histology and MTT staining to visualize viable cells. A modified MTT assay was performed as previously described $^{13}$ to extrapolate the uniformity of viable cells present within the EVPOMEs based on the $3 \times 5 \mathrm{~cm}$ EVPOME culture. The EVPOME was immersed in $10 \mathrm{~mL}$ of the medium with $1 \mathrm{~mL}$ of MTT (Roche Molecular Biochemicals, Indianapolis, IN) at $37^{\circ} \mathrm{C}$ for $3 \mathrm{~h}$. Uniformity of viable cell distribution was observed macroscopically.

\section{Optimization of thickness of AlloDerm ${ }^{\circledR}$}

Three different thicknesses of the AlloDerm, 228.6-330.2 $\mu \mathrm{m}$ (thin), 356.6 to $508.0 \mu \mathrm{m}$ (middle), and 550-1020 $\mu \mathrm{m}$ (thick) (Life Cell Cat. No. 101010, 101020, 102049), were used to fabricate three different sizes of EVPOMEs: 15, 1.75, and $0.75 \mathrm{~cm}^{2}$. EVPOMEs $\left(15 \mathrm{~cm}^{2}\right)$ cultured for $4,6,8$, and 11 days were analyzed by histology and immunohistochemistry.

\section{Histological and immunohistochemical examinations}

EVPOMEs were fixed in $10 \%$ neutral formalin. Paraffin-embedded specimens were cut into 5 - $\mu \mathrm{m}$ sections and stained with hematoxylin and eosin (HE) for histological examination. For immunohistochemistry, sections were heated in Tris-EDTA buffer $(10 \mathrm{mM}$ Tris [Sigma-Aldrich], $1 \mathrm{mM}$ EDTA [Sigma-Aldrich], $\mathrm{pH}$ 9.0) for $20 \mathrm{~min}$. After incubating the sections with 5\% normal goat serum (Fisher Scientific, Pittsburgh, PA) for $1 \mathrm{~h}$ at room temperature, they were incubated overnight at $4^{\circ} \mathrm{C}$ in a humidified chamber with primary antibodies: mouse monoclonal antibody against filaggrin (ab17808; Abcam, Cambridge, United Kingdom) (1:500), involucrin (ab68; Abcam) (1:5000), p63 (ventana, 790-4509) (no dilution), a rabbit monoclonal antibody against Ki-67 (ab16667; Abcam) (1:200), K15 (ab52816; Abcam) (1:200), and a rabbit polyclonal antibody against GLUT1 (ab14683; Abcam) (1:200). The sections were incubated with the goat anti-mouse IgG $(\mathrm{H}+\mathrm{L})$ secondary antibody, DyLight 488 conjugate (Thermo Scientific, Waltham, MA), or goat antirabbit IgG $(\mathrm{H}+\mathrm{L})$ secondary antibody, DyLight 594 conjugate (Thermo Scientific), for $90 \mathrm{~min}$ in the dark. Glass coverslips were mounted onto slides in Vectashield mounting medium with DAPI (H-1200; Vector Laboratories, Burlingame, CA) for DNA staining.

\section{Liquid diffusion pattern analysis during air-liquid interface culture}

Three different thicknesses of the AlloDerm, 228.6$330.2 \mu \mathrm{m}$ (thin), 356.6-508.0 $\mu \mathrm{m}$ (middle), and 550-1020 $\mu \mathrm{m}$ (thick), were placed onto the air-liquid interface and incubated with Ponceau S (Thermo Scientific), which is used for protein staining. They were frozen in OTC compound (Sakura Finetek, Torrance, CA) and then cut into $8-\mu \mathrm{m}$ sections by Leica CM1850 Cyrostat. The diffusion pattern was histologically observed.

\section{Glucose consumption measurement}

Large EVPOME culture supernatant aliquots were taken at all the feeding days and glucose levels were measured using the GlucCell ${ }^{\mathrm{TM}}$ (Cesco Bioengineering, Taichung, Taiwan) glucose monitoring system. Fresh medium was measured as baseline glucose, and hourly consumption of glucose was calculated by the formula as follows:

$$
\left[\left(\mathrm{G}_{\mathrm{B}}-\mathrm{G}_{\mathrm{S}}\right) / \mathrm{t}_{\mathrm{i}}\right]
$$

where $G_{B}$ is the baseline glucose amount (mg/dL), $G_{S}$ is the supernatant glucose amount (mg/dL), and $t_{i}$ is the time in hours.

\section{2-Deoxyglucose uptake assay}

To measure glucose diffusion into the AlloDerm, uptake of 2-deoxyglucose (2DG; Sigma-Aldrich) was measured. Small EVPOMEs were fabricated as described above, and glucose consumption of spent medium from days 3 to 4 was analyzed to check viability. EVPOMEs were transferred to the air-liquid interface and incubated for 3, 12, and $24 \mathrm{~h}$ with Epilife containing $6 \mathrm{mM}$ of $2 \mathrm{DG}$ as substitution for Dglucose. EVPOMEs were washed thrice with $200 \mu \mathrm{M}$ Phloretin (Sigma-Aldrich)/PBS and lysed in RIPA buffer (50 mM Tris-HCl pH7.5, $150 \mathrm{mM} \mathrm{NaCl}, 1 \%$ NP40, $0.25 \%$ Na-deoxycholate, $10 \mathrm{mg} / \mathrm{mL}$ BSA, 2 mM EGTA). Following sonication, cell lysates were collected and diluted to 1:50, and 2DG uptake was quantified by the 2-Deoxyglucose Uptake Measurement kit (Cosmo Bio, Tokyo, Japan). To eliminate variations among individual cell strains, data from the same cell strain were divided by the 3-h EVPOME of the thin AlloDerm uptake value and multiplied by 100 .

\section{Statistical analysis}

Results are presented as mean \pm standard deviation of mean (SD) of the values obtained. Comparisons among three different thicknesses were analyzed by the TukeyKramer test after determining whether the samples were normally distributed or not. A $p$-value of less than 0.05 was considered statistically significant. Statcel 3 (OMS, Tokorozawa, Japan) was used for statistical analysis.

\section{Results}

\section{Optimization of cell number for a large device}

The seeded cell density of $2.0 \times 10^{5}$ cells $/ \mathrm{cm}^{2}$, which we usually use for fabrication of up to $3 \mathrm{~cm}^{2}$ EVPOMEs resulted in a discontinuous epithelial monolayer in the large manufactured EVPOME $\left(15 \mathrm{~cm}^{2}\right)$ (Fig. 2A), while the small EVPOME $\left(0.75 \mathrm{~cm}^{2}\right)$ grew well at this cell density (Fig. 2E). The seeded cell density of $4.0 \times 10^{5}, 5.3 \times 10^{5}$, and $6.0 \times 10^{5}$ cells $/ \mathrm{cm}^{2}$ produced a continuous epithelial layer (Fig. 2B-D); however, the seeded cell density of $4.0 \times 10^{5}$ cells $/ \mathrm{cm}^{2}$ produced fewer epithelial cell layers in the large EVPOME (Fig. 2B). The epithelial layer of the small EVPOME sloughed off during processing of the paraffin block in seeded cell densities of $5.3 \times 10^{5}$ and $6.0 \times 10^{5}$ cells $/ \mathrm{cm}^{2}$ (Fig. $2 \mathrm{~F}-\mathrm{G}$ ). These data indicated that seeding cell density is not proportional to the surface area of the scaffold and can result in an incomplete stratified cell layer, as seen in Figure $2 \mathrm{~F}$ and $\mathrm{G}$.

We examined the distribution of formazan crystal-positive cells using an MTT assay to assess the epithelial coverage of large-sized EVPOME grafts macroscopically (Supplementary Fig. S1; Supplementary Data are available online at www.liebertpub.com/tec). Xu et al. (2009) ${ }^{13}$ reported that all of the formazan crystal-positive cells were 


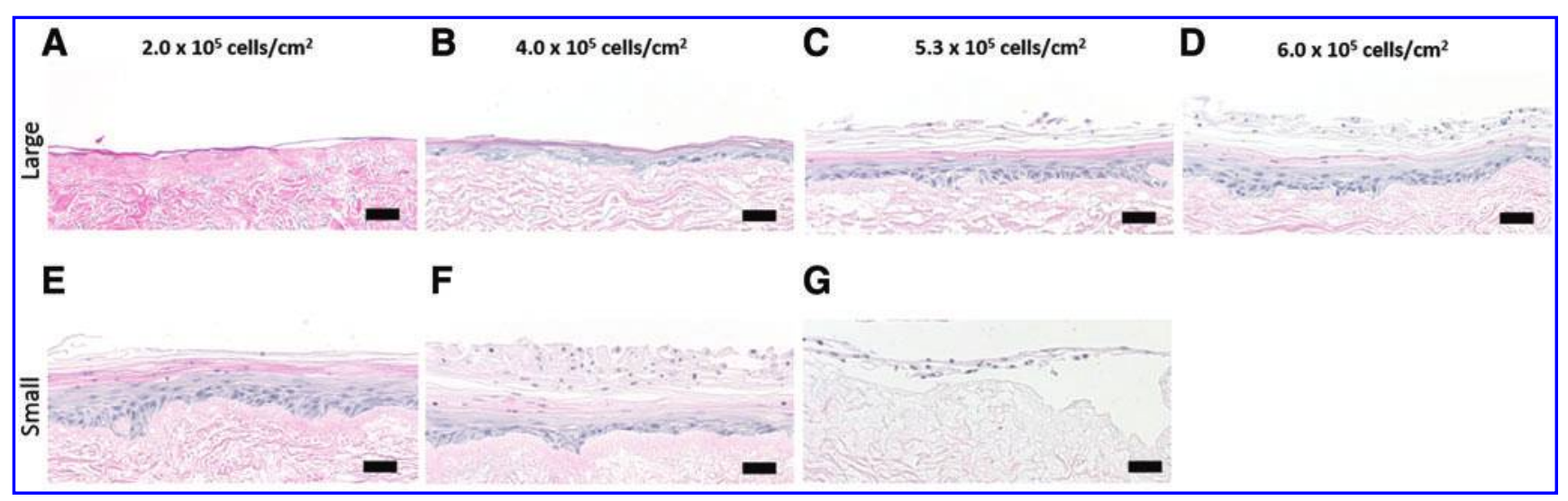

FIG. 2. Optimization of seeding density of large-sized EVPOME and comparison with small-sized EVPOME (scale $\mathrm{bar}=50 \mu \mathrm{m})$. Hematoxylin and eosin $(\mathrm{HE})$ staining of large EVPOME $\left(15 \mathrm{~cm}^{2}\right)$ with seeded cell density of $2.0 \times 10^{5}(\mathbf{A})$, $4.0 \times 10^{5}(\mathbf{B}), 5.3 \times 10^{5}(\mathbf{C})$, and $6.0 \times 10^{5}$ cells $/ \mathrm{cm}^{2}(\mathbf{D})$. Small EVPOME $\left(0.75 \mathrm{~cm}^{2}\right)$ with seeded cell density of $2.0 \times 10^{5}(\mathbf{E})$, $4.0 \times 10^{5}(\mathbf{F})$, and $5.3 \times 10^{5}$ cells $/ \mathrm{cm}^{2}(\mathbf{G})$. Color images available online at www.liebertpub.com/tec

viable and localized to the basal layer and suprabasal layer in EVPOMEs. In the macroscopic view of EVPOMEs, seeded cell densities of greater than $5.3 \times 10^{5}$ cells $/ \mathrm{cm}^{2}$ showed uniformity of viable cell distribution, while a seeded cell density of $2.0 \times 10^{5}$ cells $/ \mathrm{cm}^{2}$ showed areas of cell deficiency (Supplementary Fig. $\mathrm{S} 1 \mathrm{~A}, \mathrm{~B})$. Thus, to achieve an epithelial formation on the entire surface of a $3 \times 5 \mathrm{~cm}$ piece of AlloDerm, a minimum seeding cell density of $5.3 \times 10^{5}$ cells $/ \mathrm{cm}^{2}$ was determined and used for large EVPOME fabrication for subsequent analysis.

\section{Histological analysis of EVPOMEs of three different thicknesses and on day 11}

In the large $3 \times 5 \mathrm{~cm}$ EVPOME, HE staining of thin and middle thicknesses of AlloDerm showed well-stratified epithelium (Fig. 3A, B), while less epithelial formation was observed in the EVPOME of thick AlloDerm (Fig. 3C). However, epithelial stratification differences among three AlloDerm thicknesses were not obvious in 0.75 and $1.75 \mathrm{~cm}^{2}$ size EVPOMEs (Fig. 4D-I). Liquid diffusion pattern was visualized by ponceau $S$. The thin and thick AlloDerm had the same diffusion pattern from the bottom and edge (Fig. 4).

In immunohistochemical analysis, fewer Ki-67 (Fig. 5GI, red) and merged expression of p63 and DAPI (Fig. 5J-L, green) with lower density of basal cells were observed in the
EVPOME of thick AlloDerm (Fig. 5I, L) than EVPOMEs grown on middle and thin AlloDerm (Fig. 5G, H, J, K). Keratinocyte basal cell marker, K15 (red), and early differentiation marker, involucrin (green), were observed in all samples (Fig. 5D-F); however, a keratinocyte late differentiation marker, filaggrin, was very weak in the EVPOME grown on thick AlloDerm (Fig. 5I, green). Results of staining of all the three AlloDerm thicknesses with the size of $15 \mathrm{~cm}^{2}$ showed significant activity of GLUT1, a glucose transporter, in the thin and medium AlloDerm EVPOMEs (Fig. 5J, K, red), while very light staining was seen in the thick AlloDerm EVPOME (Fig. 5L). DAPI blue staining showed the presence of abundant nuclei in all EVPOMEs (Fig. 5A-L).

\section{Morphology and protein expression during sequential epithelial development}

Monolayer epithelial morphology was observed by $\mathrm{HE}$ staining of EVPOMEs grown on thin, medium, and thick AlloDerm on day 4 (Fig. 6A, J, S), which is the time point at which the EVPOME is airlifted for full tissue development of the device. Epithelial stratification differences in different AlloDerm thicknesses were seen over time. The epithelium development of the EVPOME grown on thick AlloDerm

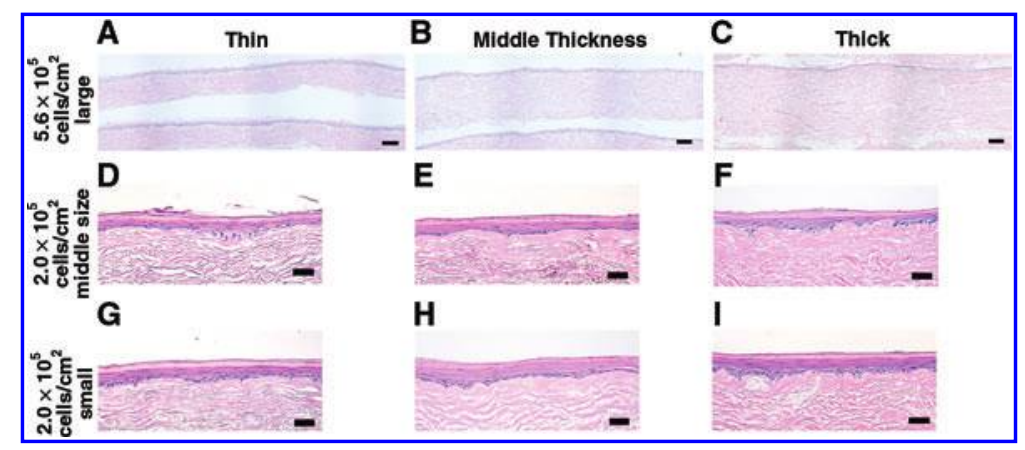

FIG. 3. HE staining of EVPOMEs of three different thicknesses and sizes $(\mathbf{A}-\mathbf{C}$, scale bar $=100 \mu \mathrm{m}$; D-I, scale bar $=$ $50 \mu \mathrm{m}$ ). EVPOME size; (A-C) 15, (D-F) 1.75, and (G-I) $0.75 \mathrm{~cm}^{2}$. AlloDerm ${ }^{\circledR}$ thickness; (A, D, G) $228.6-330.2$ (thin), (B, E, H) 355.6-508.0 (middle), and (C, F, I) 550-1020 $\mu \mathrm{m}$ (thick). Hematoxylin and eosin (HE) staining of large-sized EVPOMEs of thin and middle thicknesses of AlloDerm showed well-stratified epithelium, while less epithelial formation was observed in EVPOME of thick AlloDerm. However, epithelial stratification differences among three AlloDerm thicknesses were not obvious in 0.75 and $1.75 \mathrm{~cm}^{2}$ size EVPOMEs. Color images available online at www.liebertpub.com/tec 
FIG. 4. Liquid diffusion pattern of AlloDerm (scale bar $=200 \mu \mathrm{m})$. (A, C) Thin AlloDerm, (B, D, E) thick AlloDerm, (A, B) 8, (C, D) 48, (E) 96, and (F) $144 \mathrm{~h}$ of incubation. Both thin and thick AlloDerm showed the same pattern of diffusion from the bottom and edge. Color images available online at www liebertpub.com/tec

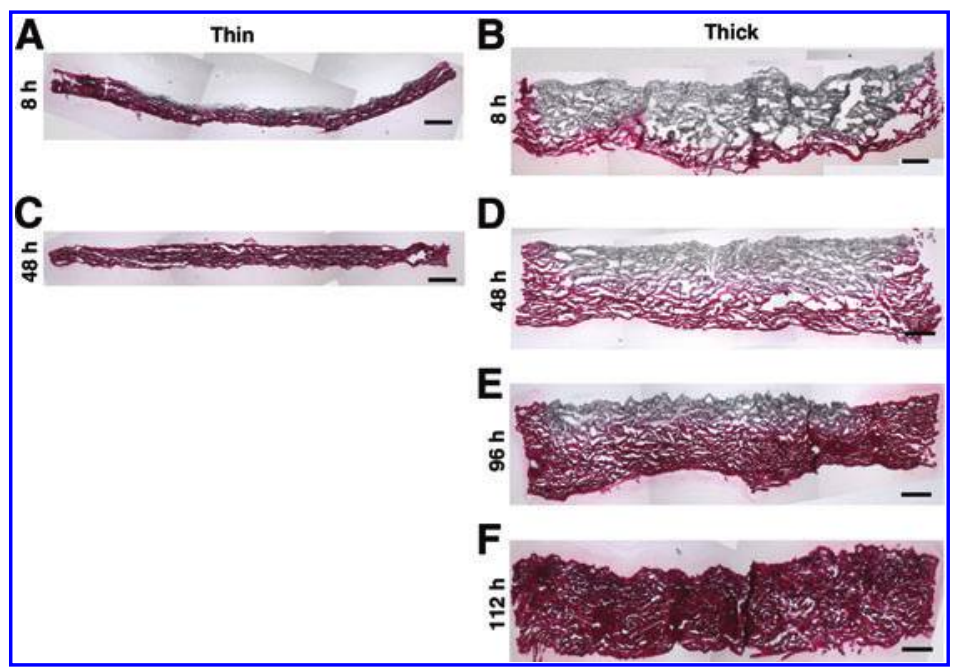

was the slowest (Fig. 6S-U). EVPOMEs of thin and middle thicknesses of AlloDerm had better stratification of the epithelium than EVPOMEs of thick AlloDerm (Fig. 6A-C, JL, S-U).

In immunohistochemical analysis, using a number of markers of differentiation and proliferation, the expression patterns of EVPOMEs of thin and middle thicknesses of AlloDerm were similar (Fig. 6D-I, M-R). In the thick AlloDerm EVPOME, less expression of Ki-67 was observed after day 6 when compared with EVPOMEs grown with thin and middle thicknesses of AlloDerm, and decreasing expression of filaggrin was seen during the entire culture period (Fig. 6D-F, M-O, V-X). p63 and GLUT1 were equally expressed on day 4 EVPOMEs in all samples (Fig. 6G, P, Y). After day 6, the expression of both p63 and GLUT1 was decreased in the EVPOME of thick AlloDerm (Fig. 6Z, AA); however, it gradually increased in thin and middle thicknesses of EVPOMEs (Fig. 6H, I, Q, R).

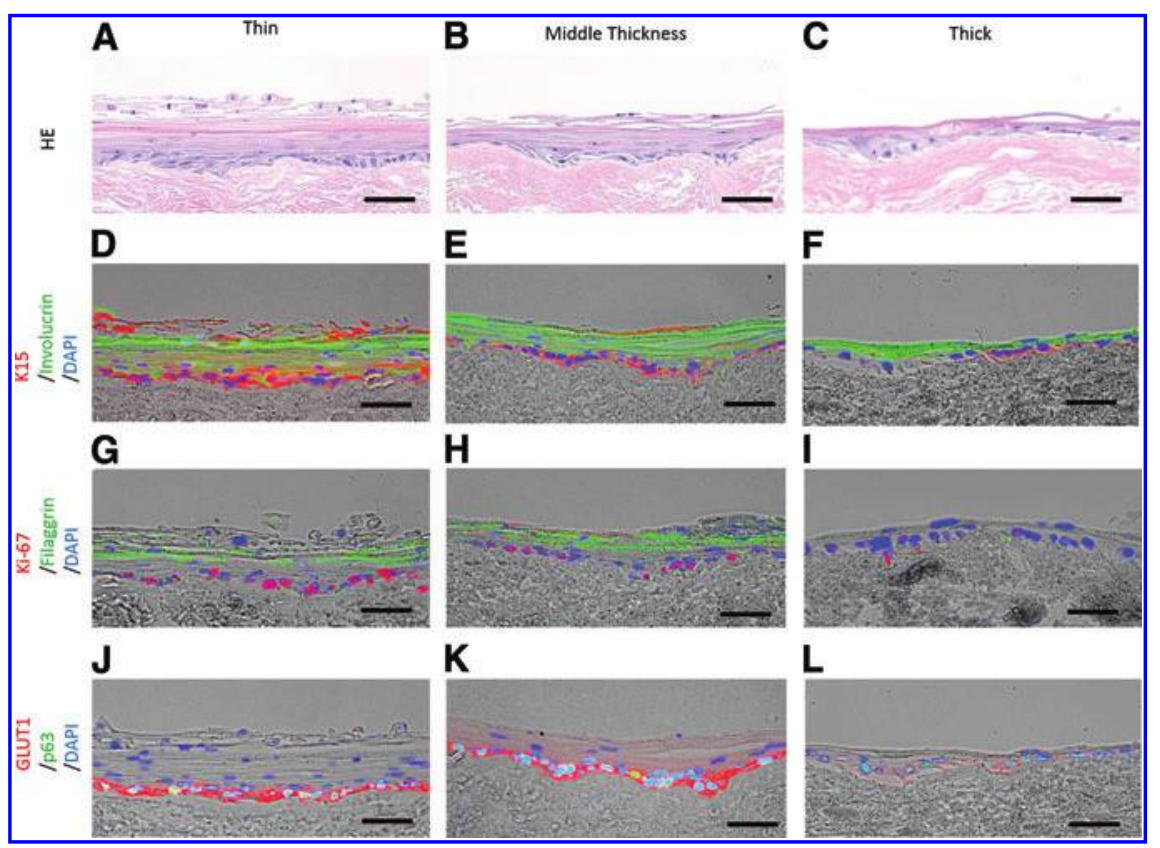

FIG. 5. Effects of AlloDerm thickness to the keratinocytes on day 11 large EVPOME characterized by immunohistochemistry (scale bar $=50 \mu \mathrm{m})$. AlloDerm thickness: $(\mathbf{A}, \mathbf{D}, \mathbf{G}, \mathbf{J})$ thin, $(\mathbf{B}, \mathbf{E}, \mathbf{H}, \mathbf{K})$ middle thickness, and $(\mathbf{C}, \mathbf{F}, \mathbf{I}, \mathbf{L})$ thick. (A-C) HE staining; EVPOME of thick AlloDerm showed decreasing epithelial stratification. (D-F) Involucrin (green), epithelial early differentiation marker, K15 ( red), epithelial basal cell marker; involucrin-expressed suprabasal layer of all samples. K15 expression was weak in EVPOME of thick AlloDerm. (G-I) Filaggrin (green), epithelial late differentiation marker, Ki-67 (red), proliferation marker; decreasing expression of filaggrin and Ki-67 was observed in EVPOME of thick AlloDerm. (J-L) p63 (green), epithelial progenitor marker, GLUT1 (red), glucose transporter; decreasing expression of p63 and GLUT1 was observed in EVPOME of thick AlloDerm. DAPI (blue) was used for DNA staining in (D-L). Color images available online at www.liebertpub.com/tec 


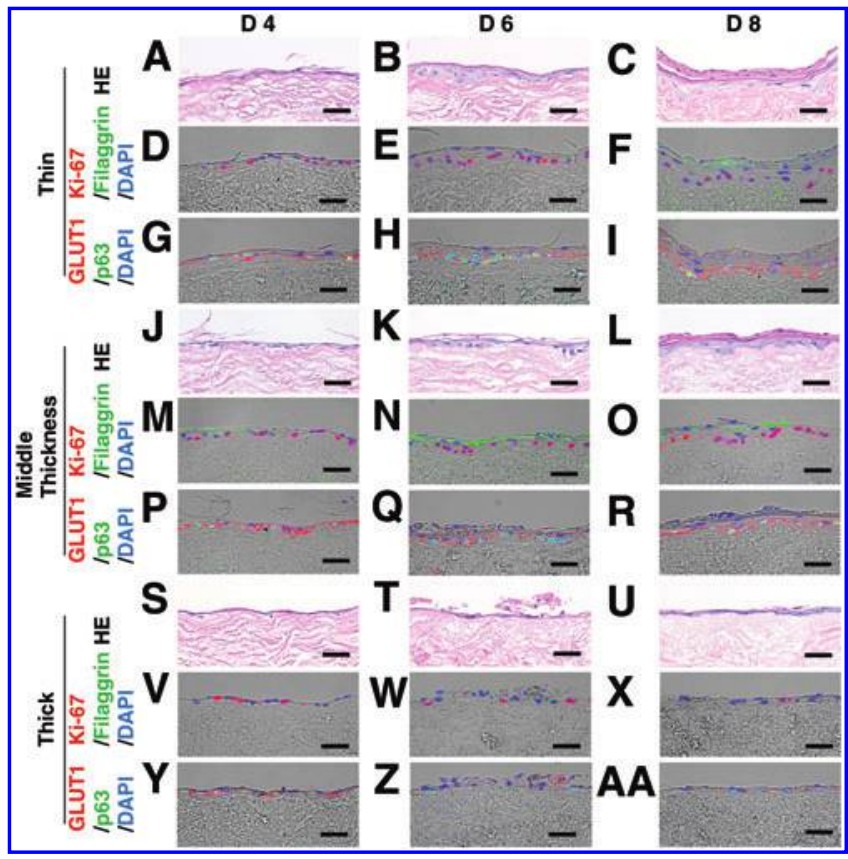

FIG. 6. Effects of AlloDerm thickness on keratinocytes during air-liquid culture of large EVPOME characterized by immunohistochemistry (scale bar $=50$ $\mu \mathrm{m})$. AlloDerm thickness: (A-I) thin, (J-R) middle thickness, and (S-AA) thick. Day of culture: $(\mathbf{A}, \mathbf{D}, \mathbf{G}$, $\mathbf{J}, \mathbf{M}, \mathbf{P}, \mathbf{S}, \mathbf{V}, \mathbf{Y})$ day $4,(\mathbf{B}, \mathbf{E}, \mathbf{H}, \mathbf{K}, \mathbf{N}, \mathbf{Q}, \mathbf{T}, \mathbf{W}$, $\mathbf{Z})$ day 6 , and $(\mathbf{C}, \mathbf{F}, \mathbf{I}, \mathbf{L}, \mathbf{O}, \mathbf{R}, \mathbf{U}, \mathbf{X}, \mathbf{A A})$ day 8 . HE staining (A, B, C, J, K, L, S, T, U). EVPOME of thick AlloDerm showed decreasing epithelial stratification $(\mathbf{S}, \mathbf{T}, \mathbf{U})$. Immunohistochemistry $(\mathbf{D}, \mathbf{E}, \mathbf{F}, \mathbf{M}$, $\mathbf{N}, \mathbf{O}, \mathbf{V}, \mathbf{W}, \mathbf{X})$. Filaggrin (green), epithelial late differentiation marker, Ki-67 (red), proliferation marker; decreasing expression of $\mathrm{Ki}-67$ was observed in EVPOME of thick AlloDerm after day $6(\mathbf{W}, \mathbf{X})$ and decreasing expression of filaggrin was observed during the entire culture period $(\mathbf{V}, \mathbf{W}, \mathbf{X})$. Immunohistochemistry $(\mathbf{G}, \mathbf{H}, \mathbf{I}, \mathbf{P}, \mathbf{Q}, \mathbf{R}, \mathbf{Y}, \mathbf{Z}, \mathbf{A A})$. p63 (green), epithelial progenitor marker, GLUT1 (red) glucose transporter; both GLUT1 and p63 expression was observed in all samples on day 4; however, gradual decreasing of their expression was observed in EVPOME of thick AlloDerm after day 6 (Z, AA). DAPI was used to stain the cell nuclei in the epithelium. Color images available online at www.liebertpub.com/tec

\section{Glucose consumption}

During submerged culture (days 0-4), all three thicknesses of EVPOMEs had similar glucose consumption. However, during air-liquid interface (days 6-11) EVPOMEs using thick AlloDerm showed lower glucose consumption than thin and middle thicknesses. Statistical differences were noted on thick AlloDerm versus thin and middle thicknesses of AlloDerm on day $10(p<0.05)$ (Fig. 7).

\section{2-Deoxyglucose uptake}

The EVPOMEs constructed using thin AlloDerm took up the greatest amount of $2 \mathrm{DG}$ at the 3 -h time point,

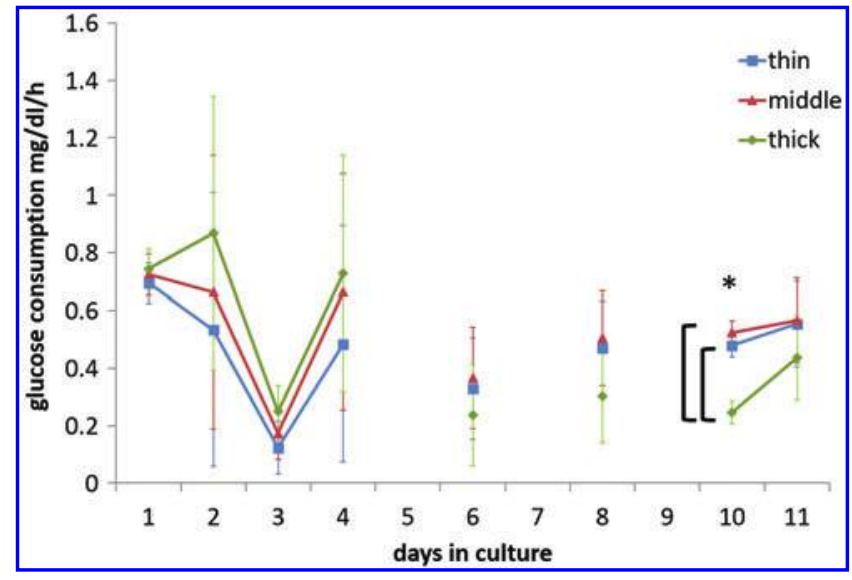

FIG. 7. Glucose consumption of large EVPOME $(N=5)$. The EVPOMEs are submerged in the medium until they are airlifted on day 4. Glucose consumption on day 10 is statistically different between EVPOME of thick AlloDerm versus thin and middle thicknesses of AlloDerm. Asterisks represent statistically significant differences determined by repeated one-way analysis of variance with the Tukey-Kramer post hoc test) $(* p<0.05)$. Color images available online at www liebertpub.com/tec

which is when the EVPOME was transferred to the airliquid interface (Fig. 8). EVPOMEs growing on middle thickness of AlloDerm showed highest uptake at $12 \mathrm{~h}$. These results would seem to indicate that $2 \mathrm{DG}$ diffused through the AlloDerm to the cells growing on the top at varying rates over time. In contrast, EVPOMEs constructed using thick AlloDerm showed lower levels of 2DG uptake. This would imply that a sufficient amount of glucose for keratinocyte growth and proliferation was not available for the cells grown on thick AlloDerm. Uptake data of all EVPOMEs at the 24-h time point showed decreasing of 2DG uptake because of cell death since 2DG is not metabolized to produce ATP in the cells. Statistical differences were noted on thin AlloDerm versus middle thickness $(p<0.05)$ and thick AlloDerm $(p<0.01)$ at $3 \mathrm{~h}$ (Fig. 8).

\section{Discussion}

To engineer large autologous tissue is a challenge. ${ }^{14}$ An equipment design of a specific shape and size and a scaffold suitable for cell proliferation and differentiation are needed. ${ }^{14}$ In addition, the fabrication protocol should be consistent with the guidelines for cell-based products developed by the Center for Biologics Evaluation and Research (CBER) of the United States Food and Drug Administration (FDA). In this study, we were able to utilize original cultureware from commercialized culture dishes, ABS plastics, and cyanoacrylate that were cell safe materials.

We investigated the optimal number of cells necessary to fabricate a large EVPOME. Our past studies indicated that a 


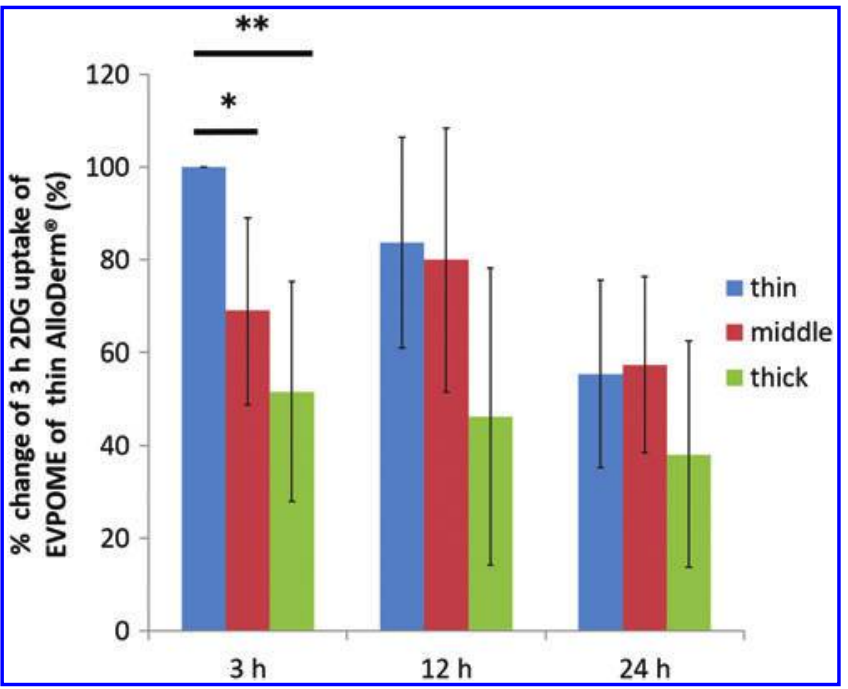

FIG. 8. 2-Deoxyglucose (2DG) uptake assay during airliquid interface culture $(N=5)$. All 2DG uptake data from the same cell strain were divided by the 3 -h value of EVPOME growing on thin AlloDerm, and \% change of 3-h uptake of EVPOME using thin AlloDerm is shown. Thus, 3-h thin AlloDerm value is shown as $100 \%$. The lower number (\%) indicates that the amount of 2DG uptake is smaller compared with that of 3-h thin AlloDerm. Asterisks represent statistically significant differences determined by repeated one-way analysis of variance with the Tukey-Kramer post hoc test) $(* p<0.05$ and $* * p<0.01)$. Color images available online at www.liebertpub.com/tec

seeding cell density of $2.0 \times 10^{5}$ cells $/ \mathrm{cm}^{2}$ was needed for up to $3.14 \mathrm{~cm}^{2}$ EVPOMEs. This cell density was not optimal to make larger $3 \times 5 \mathrm{~cm}$ EVPOMEs as shown in Figure 2. This was also confirmed by an MTT assay, which allows us to assess the epithelium coverage of the large-sized EVPOME grafts macroscopically and rapidly with low cost. This study revealed that a seeding cell density of $5.3 \times 10^{5}$ cells/ $\mathrm{cm}^{2}$ was necessary to fabricate EVPOMEs with sufficient stratification of the uppermost epithelium. It was noted that at a seeding cell density of $6.6 \times 10^{5}$ cells $/ \mathrm{cm}^{2}$, the epithelial structure showed no difference when compared with $5.3 \times 10^{5}$ cells $/ \mathrm{cm}^{2}$. This would indicate that a larger seeding cell number might not make a difference in cellular stratification after a resulting cell monolayer has been established. However, an excessive number of seeded cells might adversely affect the epithelial layer and be sloughed off as seen using a smaller size AlloDerm with a seeded cell density of $5.3 \times 10^{5}$ cells $/ \mathrm{cm}^{2}$. These results are consistent with our previous study. ${ }^{15}$

In $3 \times 5 \mathrm{~cm}$ EVPOMEs, scaffold thickness differences affected the stratification of the epithelium caused by variations in glucose diffusion through the AlloDerm during the air-liquid interface period of EVPOME construction in culture. Decreasing expression of filaggrin in EVPOMEs grown using thick AlloDerm would indicate a compromised tissue protection by the EVPOME to outside damage after transplantation onto the wound site since filaggrin plays a role in barrier function.

Glucose uptake of the EVPOME of middle thickness AlloDerm was significantly slower than the thin AlloDerm; however, it did not impact epithelial proliferation and dif- ferentiation between EVPOMEs of thin and middle thicknesses of AlloDerm. Stratification differences among the three different AlloDerm thicknesses in small and middlesized EVPOMEs were not evident. The diffusion pattern revealed by Ponceau $\mathrm{S}$ indicated that liquid diffuses from the bottom and edges when the AlloDerm is at an air-liquid interface. Thus, the smaller the size of the scaffold, the lower the effect of the diffusion would be on the viability, growth, and differentiation of the seeded cells. In contrast, the larger piece of AlloDerm showed a lower diffusion rate that affected cell viability within the central portion of the scaffold. Therefore, the slow diffusion observed in the thick and largesized AlloDerm would lead to the limited availability and a concentration gradient of other biological cues, including oxygen and growth factors regulating keratinocyte fate. ${ }^{16}$

We investigated glucose as a representative nutrition. However, it is reported that glucose permeability of deepithelialized dermis and rat keratinocytes is faster than lipophilic corticosterone, ${ }^{17}$ suggesting that the diffusion rate of other medium nutrients might also affect the stratification of keratinocytes in our culture system. Previous studies indicated that GLUT1, which is located along the basal epithelial layer, might play an important role in mediating nutrient and fluid supply to the upper layers of the epithelium from the underlying stroma. ${ }^{8,18,19}$ The reduced GLUT1 expression represents a shift from glucose-dominated to fatdominated metabolism during the differentiation process of basal cells into superficial keratinocytes. ${ }^{20,21}$ The reduction of GLUT1 expression in the EVPOME using thick AlloDerm after being raised at an air-liquid interface culture supports the finding that it consumes and metabolizes less amount of glucose because of its limited availability through diffusion through a thicker AlloDerm. In addition, the significant drop of the glucose consumption of the large EVPOME using thick AlloDerm at day 10 was correlated with the decrease in the number of Ki-67-positive cells, which may result from a decline in energy demand by mitotic activity. $^{22}$ Keratinocytes seeded in this slowed diffusion environment might be under starvation-like conditions during the air-liquid interface culture.

Under energy starvation conditions, cells restore the energy balance and protect cells from energy deprivation-dependent apoptosis. ${ }^{23} \mathrm{HaCaT}$ cells cultured in high calcium with lowglucose condition showed the progressive cell quiescence and gradual commitment into early differentiation without cell death when compared with normal glucose. ${ }^{24} \Delta$ Np63 responses of glucose starvation and TAp63 are critical regulators of lipid and glucose metabolism, regulating and integrating the metabolic response to caloric restriction and starvation. $^{25,26}$ Pan-p63 is crucial to the development of stratifying epithelia in humans and plays a multifaceted role in the mature epidermis, ${ }^{26}$ and Truong et al. $(2006)^{27}$ reported that human primary keratinocytes, in a 3D culture model using cells with knocked down pan-p63, showed decreased expression of proliferation and differentiation proteins. Our findings of glucose uptake, p63 expression, and differentiation seen in EVPOMEs of thick AlloDerm are consistent with those studies. Because of low-glucose utility, keratinocytes on the thick AlloDerm are assumed to exist in a low metabolism condition that leads to decreasing of pan-p63 expression, resulting in a diminution of cellular proliferation, stratification, and late differentiation while occurrence of 
early differentiation. There might be a critical scaffold thickness that deteriorates the quality of epithelium within the EVPOME, highly likely to be the thickness that exceeds the middle one. This study would seem to indicate that the cellular phenotype regulated by glucose metabolism during epithelial development on tissue-engineered culture is influenced by scaffold thickness, size, and medium diffusion.

In situ studies indicate that a scaffold thickness less than $1 \mathrm{~mm}$ of biomaterials encourages rapid regeneration, and grafts thicker than $1 \mathrm{~mm}$ have a higher incident of graft failure after transplantation since new blood vessels cannot grow quickly enough into the scaffold to nourish the overlaying epidermal layer. ${ }^{28,29}$ Efficient diffusion of nutrition through the dermal scaffold is a key factor to create a wellstratified EVPOME. This would also impact on wound healing after transplantation since growth factor(s) secreted by keratinocytes on the EVPOME could reach the host quicker to induce vascularization. Therefore, material thickness has direct implications on its functionality both in vitro and in vivo.

\section{Conclusion}

This study determined that a higher number of cells $\left(5.3 \times 10^{5} \mathrm{cells} / \mathrm{cm}^{2}\right)$ is necessary to seed onto the large size scaffold for the fabrication of $3 \times 5 \mathrm{~cm}\left(15 \mathrm{~cm}^{2}\right)$ EVPOME grafts that exhibit a well-stratified epithelium optimal for use in reconstruction of severe trauma, deformity, and cancer resection. Histological and immunohistochemical examinations in this study also demonstrated that the welldifferentiated and proliferative epithelial layer was developed within the EVPOME graft using AlloDerm thinner than $508.0 \mu \mathrm{m}$ in thickness when fabricating a large-sized $15 \mathrm{~cm}^{2}$ EVPOME. This was due to sufficient glucose availability for keratinocytes in the basal layer as confirmed by an increase in their glucose consumption and uptake. Thus, the scaffold size and thickness have an impact on the cellular phenotype and epithelial maturation in the manufacturing process of the EVPOME due to the glucose accessibility influenced by the diffusion rate. These outcomes provide basic strategies to manufacture a large-sized and healthy EVPOME graft for reconstructing large mucosa defects.

\section{Acknowledgments}

The authors thank Dr. Shiuhyang Kuo for his technical assistance and critical comments and the Department of Defense for providing the funding for this investigation through a subcontract to the University of Michigan from Rutgers, The State University of New Jersey: AFIRM I Contract No. W81XWH-08-2-0034.

\section{Disclosure Statement}

No competing financial interests exist.

\section{References}

1. Pogrel, M.A. Intraoral dermis grafting: has it any advantages? Oral Surg Oral Med Oral Pathol 60, 598, 1985.

2. Hillerup, S. Grafting of skin and oral mucosa in the oral and maxillofacial regions. Oral Maxillofac Clin North Am 5, $557,1993$.
3. Izumi, K., Neiva, R.F., and Feinberg, S.E. Intraoral grafting of tissue-engineered human oral mucosa. Int J Oral Maxillofac Implants 28, e295, 2013.

4. Izumi, K., Feinberg, S.E., Iida, A., and Yoshizawa, M. Intraoral grafting of an ex vivo produced oral mucosa equivalent: a preliminary report. Int J Oral Maxillofac Surg 32, 188, 2003.

5. Loh, Q.L., and Choong, C. Three-dimensional scaffolds for tissue engineering applications: role of porosity and pore size. Tissue Eng Part B Rev 19, 485, 2013.

6. Izumi, K., Song, J., and Feinberg, S.E. Development of a tissue-engineered human oral mucosa: from the bench to the bed side. Cells Tissues Organs 176, 134, 2004.

7. Spravchikov, N., Sizyakov, G., Gartsbein, M., Accili, D., Tennenbaum, T., and Wertheimer, E. Glucose effects on skin keratinocytes: implications for diabetes skin complications. Diabetes 50, 1627, 2001.

8. Terashi, H., Izumi, K., Deveci, M., Rhodes, L.M., and Marcelo, C.L. High glucose inhibits human epidermal keratinocyte proliferation for cellular studies on diabetes mellitus. Int Wound J 2, 298, 2005.

9. Moharamzadeh, K., Brook, I.M., Van Noort, R., Scutt, A.M., and Thornhill, M.H. Tissue-engineered oral mucosa: a review of the scientific literature. J Dent Res 86, 115, 2007

10. Moharamzadeh, K., Colley, H., Murdoch, C., Hearnden, V., Chai, W.L., Brook, I.M., Thornhill, M.H., and Macneil, S. Tissue-engineered oral mucosa. J Dent Res 91, 642, 2012

11. Izumi, K., Kato, H., and Feinberg, S.E. Tissue engineered oral mucosa. In: Vishwakarma, A., Sharpe, P., Shi, S., and Ramalingam, M., eds. Stem Cell Biology and Tissue Engineering in Dental Science. Waltham, MA: Academic Press/Elsevier, 2014, pp. 721-731.

12. Kato, H., Izumi, K., Saito, T., Ohnuki, H., Terada, M., Kawano, Y., Nozawa-Inoue, K., Saito, C., and Maeda, T. Distinct expression patterns and roles of aldehyde dehydrogenases in normal oral mucosa keratinocytes: differential inhibitory effects of a pharmacological inhibitor and RNAi-mediated knockdown on cellular phenotype and epithelial morphology. Histochem Cell Biol 139, 847, 2013.

13. Xu, Q., Izumi, K., Tobita, T., Nakanishi, Y., and Feinberg, S.E. Constitutive release of cytokines by human oral keratinocytes in an organotypic culture. J Oral Maxillofac Surg 67, 1256, 2009.

14. Eiselt, P., Kim, B.S., Chacko, B., Isenberg, B., Peters, M.C., Greene, K.G., Roland, W.D., Loebsack, A.B., Burg, K.J., Culberson, C., Halberstadt, C.R., Holder, W.D., and Mooney, D.J. Development of technologies aiding largetissue engineering. Biotechnol Prog 14, 134, 1998.

15. Izumi, K., Takacs, G., Terashi, H., and Feinberg, S.E. Ex vivo development of a composite human oral mucosal equivalent. J Oral Maxillofac Surg 57, 571, 1999.

16. Kinney, M.A., Hookway, T.A., Wang, Y., and McDevitt, T.C. Engineering three-dimensional stem cell morphogenesis for the development of tissue models and scalable regenerative therapeutics. Ann Biomed Eng 42, 352, 2014.

17. Pappinen, S., Tikkinen, S., Pasonen-Seppänen, S., Murtomäki, L., Suhonen, M., and Urtti, A. Rat epidermal keratinocyte organotypic culture (ROC) compared to human cadaver skin: the effect of skin permeation enhancers. Eur J Pharm Sci 30, 240, 2007.

18. Gherzi, R., Melioli, G., Luca, M.D., D’Agostino, A., Guastella, M., Traverso, C.E., D’Anna, F., Franzi, A.T., 
and Cancedda, R. High expression levels of "erythroid/ brain" type glucose transporter (GLUT1) in the basal cells of human eye conjunctiva and oral mucosa reconstituted in culture. Exp Cell Res 195, 230, 1991.

19. Yoshizawa, M., Feinberg, S.E., Marcelo, C.L., and Elner, V.M. Ex vivo produced human conjunctiva and oral mucosa equivalents grown in a serum-free culture system. $\mathbf{J}$ Oral Maxillofac Surg 62, 980, 2004.

20. Freinkel, R.K. Carbohydrate metabolism of epidermis. In: Goldsmith, L.A. eds. Biochemistry and Physiology of the Skin (2nd Edition). New York, NY: Oxford University Press, 1991, pp. 452-461.

21. Kuroki, S., Yokoo, S., Terashi, H., Hasegawa, M., and Komori, T. Epithelialization in oral mucous wound healing in terms of energy metabolism. Kobe J Med Sci 5, E5, 2009.

22. Vander Heiden, M.G., Cantley, L.C., and Thompson, C.B. Understanding the Warburg effect: the metabolic requirements of cell proliferation. Science 324, 1029, 2009.

23. Murayama, A., Ohmori, K., Fujimura, A., Minami, H., Yasuzawa-Tanaka, K., Kuroda, T., Oie, S., Daitoku, H., Okuwaki, M., Nagata, K., Fukamizu, A., Kimura, K., Shimizu, T., and Yanagisawa J. Epigenetic control of rDNA loci in response to intracellular energy status. Cell 133, 627, 2008.

24. Aymard, E., Barruche, V., Naves, T., Bordes, S., Closs, B., Verdier, M., and Ratinaud, M.H. Autophagy in human keratinocytes: an early step of the differentiation? Exp Dermatol 20, 263, 2011.

25. Su, X., Gi, Y.J., Chakravarti, D., Chan, I.L., Zhang, A., Xia, X., Tsai, K.Y., and Flores, E.R. TAp63 is a master tran- scriptional regulator of lipid and glucose metabolism. Cell Metab 16, 511, 2012.

26. Rinne, T., Brunner, H.G., and van Bokhoven, H. p63associated disorders. Cell Cycle 6, 262, 2007.

27. Truong, A.B., Kretz, M., Ridky, T.W., Kimmel, R. and Khavari, P.A. p63 regulates proliferation and differentiation of developmentally mature keratinocytes. Genes Dev 20, 3185, 2006.

28. Berthod, F., Saintigny, G., Chretien, F., Hayek, D., Collombel, C., and Damour, O. Optimization of thickness, pore size and mechanical properties of a biomaterial designed for deep burn coverage. Clin Mater 15, 259, 1994.

29. Sahota, P.S., Burn, J.L., Heaton, M., Freedlander, E., Suvarna, S.K., Brown, N.J., and Mac Neil, S. Development of a reconstructed human skin model for angiogenesis. Wound Repair Regen 11, 275, 2003.

Address correspondence to: Stephen E. Feinberg, DDS, MS, PhD Department of Oral and Maxillofacial Surgery University of Michigan Health System Towsley Center Rm G1114 1515 East Medical Center Drive Ann Arbor, MI 48109-5222

E-mail: sefein@med.umich.edu

Received: October 25, 2014 Accepted: February 3, 2015

Online Publication Date: April 13, 2015 


\section{This article has been cited by:}

1. Shiuhyang Kuo, Hyungjin Myra Kim, Zhifa Wang, Eve L. Bingham, Atsuko Miyazawa, Cynthia L. Marcelo, Stephen E. Feinberg. 2017. Comparison of two decellularized dermal equivalents. Journal of Tissue Engineering and Regenerative Medicine 83. . [Crossref]

2. Fernanda G. Basso, Taisa N. Pansani, Diana G. Soares, Josimeri Hebling, Carlos Alberto de Souza Costa. 2017. LLLT Effects on Oral Keratinocytes in an Organotypic 3D Model. Photochemistry and Photobiology 24. . [Crossref]

3. F.G. Basso, J. Hebling, C.L. Marcelo, C.A. de Souza Costa, S.E. Feinberg. 2017. Development of an oral mucosa equivalent using a porcine dermal matrix. British Journal of Oral and Maxillofacial Surgery 55:3, 308-311. [Crossref]

4. Gaurav Shah, Bernard J. Costello. 2017. Soft Tissue Regeneration Incorporating 3-Dimensional Biomimetic Scaffolds. Oral and Maxillofacial Surgery Clinics of North America 29:1, 9-18. [Crossref]

5. Keyvan Moharamzadeh. Oral mucosa tissue engineering 223-244. [Crossref]

6. Thafar Almela, Ian M. Brook, Keyvan Moharamzadeh. 2016. Development of three-dimensional tissue engineered bone-oral mucosal composite models. Journal of Materials Science: Materials in Medicine 27:4. . [Crossref]

7. Joseph E. Cillo, David Basi, Zachary Peacock, Tara Aghaloo, Gary Bouloux, Thomas Dodson, Sean P. Edwards, Deepak Kademani. 2016. Proceedings of the American Association of Oral and Maxillofacial Surgeons 2015 Research Summit. Journal of Oral and Maxillofacial Surgery 74:3, 429-437. [Crossref] 